\title{
The Effects of Localized Trap Energy on the Photoluminescence Intensity of Gallium Nitrate (GaN) Compound Semiconductor for Different Radiative Trap Level
}

\author{
Getu Endale \\ Department of Physics, CNCS, Wolkite University, P.O.Box 07, Wolkite, Ethiopia
}

Received August 13, 2019; Revised September 19, 2019; Accepted September 25, 2019

Copyright $\odot 2019$ by authors, all rights reserved. Authors agree that this article remains permanently open access under the terms of the Creative Commons Attribution License 4.0 International License

\begin{abstract}
In this paper, we model effects of localized trap energy on the photoluminescence at different radiative trap level. Wherever possible, the concepts are augmented with data, with particular emphasis in the case of Gallium nitrate. By using illumination and lifetime, the intensity of light in each band is determined by assuming one incident photon ejects one electron at a time. From this at different temperature, illumination, doping concentration and impurity densities of states the intensities of light vary for all radia-tive recombination mechanisms. By varying illumination and impurity densities of states at room temperature, the dominated radiative recombination mechanisms are studied from the three radiative recombination mechanisms. At high values of illumination, the intensity of light in band-to-band radiative recombination mechanism dominates for all values of localized trap energies. For high values of impurity trap density, only the intensity of light in conduction band to trap level radiative recombination mechanisms dominates for all localized trap energies.
\end{abstract}

Keywords Photoluminescence, Gallium Nitrate, Doping, Injection Level, Radiative Recombination, Trap Energy

\section{Introduction}

The abundance of gallium in the Earth's crust is small, generally less than 19 parts per million. Gallium does not exist in elemental form in nature, and the few high-gallium minerals that do occur are too rare to serve as a primary source of the element. Fortunately, gallium occurs in low amounts in many minerals where it substitutes for elements of similar size and charge. Gallium is found in small amounts ( 50 parts per mil- lion) in many bauxite deposits, and it occurs in zinc deposits in about the same amounts as in bauxite deposits. Currently, gallium is obtained mainly from mining and mineral processing of bauxite ore for aluminum, although some gallium is also derived from the processing of sphalerite ore for zinc. Gallium is also recycled from scrap generated in the manufacture of GaAs- and GaN-based devices [1].

The GaN compound semiconductor have had a surprisingly long history despite the recent increase in activities of LED and lasers emitting in the blue and ultraviolet (UV) wavelengths and Devices capable of operation at high temperatures, i.e. $>300$. Until recently the vast majority of semiconductor optical devices operate from IR to green wavelengths. If this range could be extended into the blue and UV region of the electromagnetic spectrum, the ability to generate the three primary colors would allow a range of display applications to become viable. In addition, the shorter wavelengths would allow increased densities for data storage. The wurtzite poly types of $\mathrm{GaN}$ form a continuous alloy system whose direct band gap is $3.4 \mathrm{eV}$ for $\mathrm{GaN}$, This potential for fabricating optical devices has recently been realised and is progressing rapidly although there remain areas of material and device physics that are still controversial [2]. The large band gap means that the associated intrinsic carrier density is small. The intrinsic carrier density of $\mathrm{GaN}$ at 1300 is $10^{15} \mathrm{~cm}^{-3}$. This makes GaN suitable for applications in high temperature environments, such as in car engines, and in devices where self-heating causes the device temperature to rise. Such systems normally require sophisticated cooling which adds both to the cost and the weight. However, the temperature dependence of the hole carrier density currently observed is likely to limit bipolar device operation [3].

Semiconductor device performance depends upon the controllability of the electrical properties of the constituent materials. In $\mathrm{GaN}$, even in the absence of any intentional doping and independent of the growth technique, a high back- 
ground electron concentration $\left(\geq 10^{17} \mathrm{~cm}^{-3}\right)$ has been reported [4]. Recently reported that, the $\mathrm{N}$ vacancy is one of the major sources of carriers when the carrier concentration $\left(n<2 \times 10^{17} \mathrm{~cm}^{-3}\right)$, but the main source should be other defects when $\left(n>2 \times 10^{17} \mathrm{~cm}^{-3}\right)$. In order to overcome the large lattice mismatch they grew thick $(>100 \mu m)$ GaN layers on sapphire. By this approach they succeed in reducing the background concentration from $10^{19} \mathrm{~cm}^{-3}$ to $10^{17} \mathrm{~cm}^{-3}$. They performed temperature dependent Hall measurements from $10 \mathrm{~K}$ to $900 \mathrm{~K}$ on $\mathrm{GaN}$ having a room temperature background electron concentration $\left(n=3 \times 10^{17} \mathrm{~cm}^{-3}\right)$. However, it should be noted that due to the range of edge dislocation densities in such epilayers the non-uniformity makes interpretation of the transport measurements more difficult [5].

Gallium nitride $(\mathrm{GaN})$ is a semiconductor compound expected to make possible miniaturized, high-power wireless transmitters. These transmitters will be combined with sensitive receivers into telephone sets capable of directly accessing communications satellite. The compound can also be used in light-emitting diodes (LED s) and other semiconductor devices. The advantages of GaN devices include high output power with small physical volume, and high efficiency in power amplifiers at ultra-high and microwave radio frequencies. The main problem with $\mathrm{GaN}$ technology is cost. A special process is required to grow a GaN crystal or wafer on which transistor s and integrated circuits (IC's) can be fabricated. Once the process is implemented on a large scale, the cost should come down [6].

Gallium Nitride is so called third generation semiconductor materials with wide band gap. With the rapid developments of semiconductor technology, it has become the research focus in semiconductor materials in past decades. The band gap of GaN is $3.39 \mathrm{eV}$ at room temperature. Particularly, the GaN-based materials have the advantages of wide band gap, fast electron drift velocity, high temperature resistance and high pressure tolerance. So they have attracted much attention in the preparation of short wavelength luminescence device and high power microwave device. GaN is the core material and basic device of the new semiconductor opto-electronics industry. The defects and impurities in GaN have a great influence on the electron transport and optical properties of materials [7].

The formation energy of the positively (negatively) charged defects increases (decreases) as the Fermi level moves from the valence-band maximum towards the conduction band. The slope of this variation is proportional to the charge state of the defect. The energy at which the levels corresponding to different charge states intersect determines the ionization level of the defect. self-interstitial and antisite defects have very high formation energies and thus are unlikely to occur in GaN during growth at least in n-type GaN. Gallium and nitrogen vacancies may be abundant in n- and p-type $\mathrm{GaN}$, respectively. It must be noted, however, that electron irradiation or ion implantation can create the defects which have high formation energy in large concentrations [8].

Thermal energy induces a vibrational motion to the atoms around their equilibrium positions. Consequently, as the band structure is calculated in an idealised static model, a change in the band structure with temperature is expected. Temperature can affect the band structure by two mechanisms, the thermal expansion of the lattice and the electron-phonon interaction. The former is the dominant mechanism at low temperatures while the phonon density is small. Theoretical analysis shows that the band gap of semiconductors in general decreases as the temperature increases [9].

For different semiconductor devices, one needs materials with different parameters, like energy band-gap. Physical properties are very different among different semiconductors due to distinct characteristics of energy band-gaps and impurities. These impurities play a major role in determining the electrical and optical properties of semiconductors [10]. Almost all of today's technology involves the use of semiconductors, with the most significant aspect being the integrated circuit (IC). One of the most commonly used techniques to investigate properties in semiconductors is Photoluminescence (PL). PL has become a standard method for the characterization of semiconductors properties. It can be used to determine energy levels, concentration of impurities, defects and fundamental properties of semiconductors. One of the most common examples of photoluminescence is the fluorescent lamp [11].

In this work, a different factor that affects the Photoluminesce intensity of Gallium nitrate $(\mathrm{GaN})$ compound semiconductor for different radiative trap level were studied. These can be done by varying doping level, localized trap energy, trap density and illumination.

The remaining parts of this work is presented as follows: In the remainder of this section a brief theoretical background is presented. Then, result and discussion is presented. Finally, summary and conclusion of this study was described.

\section{Theory}

\subsection{Density of carries concentration in semicon- ductors}

The total thermal equilibrium density of electrons and holes in a semiconductor is given by [12]:

$$
\begin{aligned}
& n_{0}=N_{c} \exp \left(-\frac{E_{c}-E_{F}}{k_{B} T}\right) \\
& p_{0}=N_{v} \exp \left(-\frac{E_{F}-E_{v}}{k_{B} T}\right)
\end{aligned}
$$

Where,

$$
N_{c}=2{\frac{\left(2 \pi m_{n}^{*} k_{B} T\right)^{\frac{3}{2}}}{h^{2}}}^{2}
$$

And

$$
N_{v}=2{\frac{\left(2 \pi m_{p}^{*} k_{B} T\right)^{\frac{3}{2}}}{h^{2}}}^{2}
$$

Where $N_{c}$ and $N_{v}$ are the effective density of the conduction band states and effective density of the valence band states respectively, $m_{n}^{*}$ is effective mass of electrons in the conduction band and $m_{p}^{*}$ is effective mass of holes in the valence band. The values of $m_{p}^{*}$ and $m_{n}^{*}$ for $\mathrm{GaN}$ is given by [13]: 


$$
m_{p}^{*}=0.33 m_{o} \quad \text { and } \quad m_{n}^{*}=0.13 m_{o}
$$

Where, $m_{o}$ is the rest mass of electron.

Suppose that $\Re$ is the concentration of electron-hole pairs thermally generated in the respective bands and $\Re_{R}$ is the concentration of electron hole annihilated after the generation, the free carrier concentrations in the conduction and valence bands adjust for the intrinsic semiconductor to be:

$$
n_{0}=\Re-\Re_{R}=n_{i}=p_{0} .
$$

The intrinsic carrier concentrations are given as follows:

$$
n_{i}=N_{c} \exp \left(-\frac{E_{c}-E_{i}}{k_{B} T}\right)=N_{v} \exp \left(-\frac{E_{i}-E_{v}}{k_{B} T}\right)
$$

where, $E_{F}$ is the Fermi level, $E_{i}$ intrinsic energy level and $K_{B}$ Boltzmann constant.

The intrinsic Fermi energy derived from effective density of electron and holes as follows:

$$
\begin{gathered}
E_{i}=\left(\frac{E_{c}-E_{v}}{2}\right)+\frac{K_{B} T}{2} \ln \left(\frac{N_{v}}{N_{c}}\right) \\
E_{i}=\left(\frac{E_{v}+E_{c}}{2}\right)+\frac{3 K_{B} T}{2} \ln \left(\frac{m_{p}^{*}}{m_{n}^{*}}\right)
\end{gathered}
$$

which indicates that if $m_{p}^{*}=m_{n}^{*}$ or $\mathrm{T}=0$ the Fermi level in an intrinsicsemiconductor is positioned at mid-gap. In real cases $m_{p}^{*}$ different from $m_{n}^{*}$, resulting in small deviation of the fermi level from mid-gap.

The equilibrium density of electrons and holes in a nondegenerate semiconductor is constant at a given temperature and given by: [14].

$$
\begin{gathered}
n_{i}^{2}=p_{0} n_{0} \\
n_{i}=\sqrt{N_{c} N_{v}} \exp \left(\frac{E_{v}-E_{c}}{2 K_{B} T}\right)
\end{gathered}
$$

Adding donor or acceptor impurity atoms into semiconductor will change the distribution of electrons and holes in the material, and as a result, the Fermi energy will change.

$$
p_{0}=N_{i} \exp \left(-\frac{E_{i}-E_{F}}{k_{B} T}\right) \text { and } n_{0}=N_{i} \exp \left(-\frac{E_{F}-E_{i}}{k_{B} T}\right)
$$

As the charge carrier concentrations change, the position of the extrinsic Fermi energy level varies within the band gap until the system reaches thermal equilibrium. Once $m_{p}^{*}, m_{n}^{*}$ and $E_{g}$ are known at a given temperature, one can easily compute the values of $n_{i}$ and $E_{i}$. Figure 1 below illustrates the carrier density and energy band structure for intrinsic semiconductor $\left(m_{p}^{*}>m_{n}^{*}\right)$ at (a) zero T, (b) intermediate T and (d) high T. The intrinsic carrier density increases with increasing temperature.

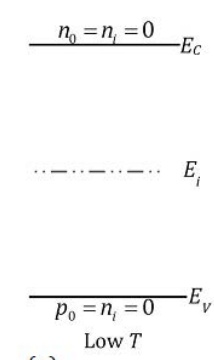

(a)

Figure 1. Carriers density and energy band structure for intrinsic semiconductor at (a) low T, (b) intermediate $\mathrm{T}$ and (d) high $\mathrm{T}$.

\subsection{Statistics of Donors and Acceptors in semi- conductors}

The density of electrons in a shallow-donor state is given by [15]:

$$
n_{d}=\frac{N_{d}}{1+\frac{1}{g_{d}} \exp \left(-\frac{E_{d}-E_{F}}{k_{B} T}\right)}
$$

The concentration of ionized donors and acceptors are given by:

$$
\begin{aligned}
& N_{d}^{+}=N_{d}-n_{d}=\frac{N_{d}}{1+g_{d} \exp \left(-\frac{E_{F}-E_{d}}{k_{B} T}\right)} \\
& N_{a}^{-}=N_{a}-n_{a}=\frac{N_{a}}{1+g_{a} \exp \left(-\frac{E_{F}-E_{d}}{k_{B} T}\right)}
\end{aligned}
$$

where $n_{d}$ is the density of electrons occupying the donor level, $N_{d}$ is the concentration of donor atoms, $E_{d}$ is the energy of the donor level and $g_{d}$ is the donor-site degeneracy factor, $p_{a}$ is the density of holes bound to acceptors, $N_{a}$ the concentration of acceptor atoms, $E_{a}$ the energy of the acceptor level relative to the valence band, $N_{a}^{-}$the concentration of ionized acceptor atoms and $g_{a}$ is the acceptor-site degeneracy factor. Most authors set $g_{d}=2$ to account for the spin degeneracy at the donor sites (only one parabolic band at the conduction band edge). It is likewise standard practice to argue that the acceptor sites must additionally reflect the two fold (heavy- and light-hole) degeneracy of the valence band. Thus, the acceptor degeneracy factor is $g_{a}=2 \times 2=4$.

According to the principle of detailed balance $\left(R_{t h}=G_{t h}\right)$, the probability of capture and emission for each carrier is equal, so that we have [16]

$$
U_{c n}=U_{e n} \quad \text { and } \quad U_{c p}=U_{e p}
$$

where

$$
e_{n}=C_{n T} n_{1} \quad \text { and } \quad e_{p}=C_{p T} p_{1}
$$

for,

$$
n_{1}=n_{i} \exp \left(\frac{E_{T}-E_{i}}{K_{B} T}\right) \quad \text { and } \quad p_{1}=n_{i} \exp \left(\frac{E_{i}-E_{T}}{K_{B} T}\right)
$$

are the concentrations of electrons and holes in the conduction and valence bands for the case in which the Fermi-level $E_{F}$ falls at $E_{T}$. 
The fraction of photo-generated electrons $I_{n}$ and holes $I_{p}$ are given by [17]:

$$
I_{n}=\frac{\delta n_{T}}{\delta n}=\frac{\tau_{p 0} p_{0 T}-\tau_{n 0} n_{0 T}}{\tau_{n 0}\left(p_{0}+p_{1}+n_{0 T}+\delta N\right)+\tau_{p 0}\left(n_{0}+n_{1}+\delta N\right)}
$$

$$
I_{p}=\frac{\delta p_{T}}{\delta p}=\frac{\tau_{n 0} n_{0 T}-\tau_{p 0} p_{0 T}}{\tau_{n 0}\left(p_{0}+p_{1}+\delta P\right)+\tau_{p 0}\left(n_{0}+n_{1}+p_{0 T}+\delta P\right)}
$$

where $\delta N$ is the total excess electron concentration generated in the conduction band, $\tau_{n 0}$ and $\tau_{p 0}$ are time constants for a given center, $\delta P$ is the total excess hole concentration generated in the valence band. The subscripts $n$ and $p$ represent the electrons and holes concentration.

The total band-to-band radiative recombination rate $R_{C V}$ [18].

$$
R_{C V}=C_{R} n p
$$

where $C_{R}$ is a constant known as the coefficient of holes.

The band to band photoluminescence effect $I_{C V}$ is given by:

$$
I_{C V}=\frac{U_{R}}{G_{0}}=\frac{C_{R}\left(p_{0} \delta n+n_{0} \delta p+\delta n \delta p\right)}{G_{0}}
$$

The conduction band to localized center radiative recombination rate $R_{C T}$ is:

$$
R_{C T}=C_{n T} n p_{T}
$$

where $C_{n T}$ is a constant known as the coefficient of holes.

The conduction band to localized state photoluminescence effect $I_{C T}$ is given by:

$$
I_{C T}=\frac{U_{C T}}{G_{0}}=\frac{C_{C T}\left(p_{0 T} \delta n+n_{0} I_{p} \delta p+I_{p} \delta p \delta n\right)}{G_{0}}
$$

The localized state to valence band radiative recombination rate $R_{T V}$ is.

$$
R_{T V}=C_{T V} p n_{T}
$$

where, $C_{T V}$ is a constant known as the coefficient of holes.

The intensity of light from the trap level to the valence band, $I_{T V}$ is given by:

$$
I_{T V}=\frac{U_{T V}}{G_{0}}=\frac{C_{T V}\left(n_{0 T} \delta p+p_{0} I_{n} \delta n+I_{n} \delta p \delta n\right)}{G_{0}}
$$

\section{Numerical Result and Discussion}

\subsection{Variation of photoluminescence with local- ized state energy with various electronic transition level}

\subsubsection{The case of fixed doping level and temperature and varying localized state densities}

The trapping of electrons by the localized state removes the same amount of $p_{T}$ states. Trapping of electrons by the trap center will reduce the concentration of excess electrons in the conduction band, so that the probability of holes' recombination with conduction band electrons is reduced. This enhances the concentration of excess holes in the valence band. The rate at which a carrier moves into the energy level in the forbidden gap depends on the distance of the introduced energy level from either of the band edges. Therefore, if an energy is introduced close to either band edge, recombination is less likely as the electron is likely to be re-emitted to the conduction band edge rather than recombine with holes, which moves into the same energy state from the valence band. For this reason, energy levels near mid-gap are very effective for recombination. Lifetime depends inversely on the density of recombination centers and capture cross sections but does not depend directly on the energy level of the impurity. It does depend indirectly on the energy level, because the capture cross section tends to be highest for impurities with near the center of the band gap and lowest for near the conduction or valence band.

As impurity density of trap states increases, the thermal equilibrium concentration of holes and electrons at trap level, the majority carrier traps in the acceptor level and the minority carrier traps in the donor level increases. The minority carrier traps further away above the intrinsic level become more effective with increasing impurity density of traps. The energy level measured relative to the minority carrier is equal to the energy difference between the Fermi level and the majoritycarrier band edge. Defect levels with energies less than this energy value have a greater probability for emitting a trapped minority carrier back to the minority carrier band than for capturing a majority carrier to complete a recombination.

The effects of traps are zero in the conduction band to valence band radiative recombination mechanism. It increases excess concentration of electrons and holes in the conduction and valence band respectively. As the impurity density of trap of state increases, the excess concentration of charge carriers' decreases. This decreases the rate of recombination and hence decreases the intensity of light. In the conduction band to trap level radiative recombination mechanism electrons from conduction band and holes from valence band recombine at localized Centre. If the impurity density of states increases, the holes' capture rate and the electron emission rate also increases. These greatly increases the recombination rates and hence increases the intensity of light in the bands.

In the localized trap state to valence band radiative recombination mechanism electrons from trap level and holes in the valence band recombine. Due to the effects of trap, only small amount of electrons on localized Centre and excess of holes in the valence band. As impurity density of states increases, more electrons recombine with holes. This increases the recombination rates and hence increases the intensity of light in the band.

Figure 2 shows the Variation of photoluminescence intensities due to various electronic transition levels with localized energies at room temperature in the $G a N$, when the localized state densities are adjusted to (a) $10^{15} \mathrm{~cm}^{-3}$, (b) $10^{17} \mathrm{~cm}^{-3}$ and (c) $10^{19} \mathrm{~cm}^{-3}$. It was drawn by using illumination $G_{0}=$ $1 \times 10^{25} \mathrm{~cm}^{-3} \mathrm{~s}^{-1}, N_{a}=1 \times 10^{17} \mathrm{~cm}^{-3}$ and $E_{a}=0.01 \mathrm{eV}$ at different impurity density of trap.

Figure 2 (a) depicts that the Variation of photoluminescence 
intensities due to various electronic transition levels with localized energies in the $G a N$, when the localized state densities are adjusted to $10^{15} \mathrm{~cm}^{-3}$. For the increasing intensity of light in the conduction band to valence band radiative recombination there is impurity density of trap states decreases. It starts to increase from $10^{4}$ to $10^{2}$ and dominates for all energy values. The intensity of light in the conduction band to trap level radiative recombination remains constant for all energies. The intensity of light in the trap level to valence band radiative recombination decreases at low energies due to holes' trap effect and remains constant up to $3.2 \mathrm{eV}$ values of localized trap energies. Then, it decreases for the remaining energies.

Figure 2 (b) shows that the Variation of photoluminescence intensities due to various electronic transition levels with localized energies in the $G a N$, for the localized state densities of $10^{17} \mathrm{~cm}^{-3}$. The intensity of light in band-to-band radiative recombination $I_{C V}$ increase from $10^{4}$ to $10^{-1]}$ and dominates only for low values of energies up to $2 \mathrm{eV}$. Above $3.2 \mathrm{eV}$ it starts to increase because of electrons in the conduction bands becomes ionized donors. The intensity of light in Conduction band to localized trap level radiative recombination constant at low temperatures and Start to decrease from $2 \mathrm{eV}-3.2 \mathrm{eV}$. and dominates for these values of energies. The intensity of light in localized trap level to Valence band radiative recombination decreases up to $0.2 \mathrm{eV}$ localized trap energy and decreases for the remaining energy.

Figure 2 (c) shows that the Variation of photoluminescence intensities due to various electronic transition levels with localized energies in the GaN, for the localized state densities of $10^{17} \mathrm{~cm}^{-3}$. The intensity of light in conduction band to trap level radiative recombination increase from $10^{4}$ to $10^{-3}$ and dominates only at very low energies. The intensity of light in Conduction band to localized trap level radiative recombination from $10^{0}$ to $10^{2}$ at low energy and decrease up to higher energies due to electron trap Centre. At energies all electrons in the conduction bands becomes ionized donor and these increases the intensity. The intensity of light in localized trap level to Valence band radiative recombination decreases from $10^{2}$ to $10^{1}$. It decreases starting from $0.5 \mathrm{eV}$ values of localized trap energies.

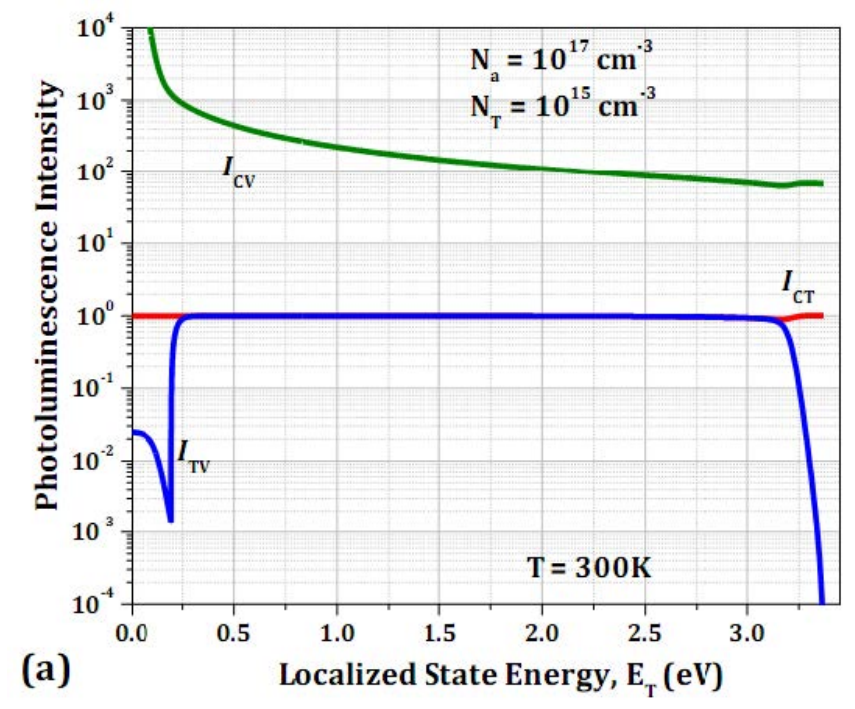

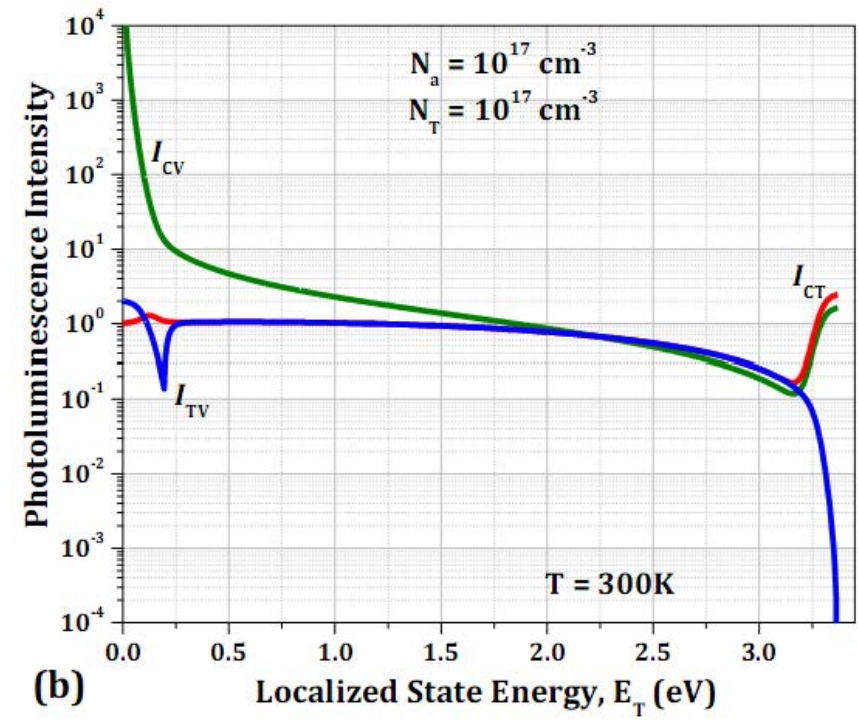

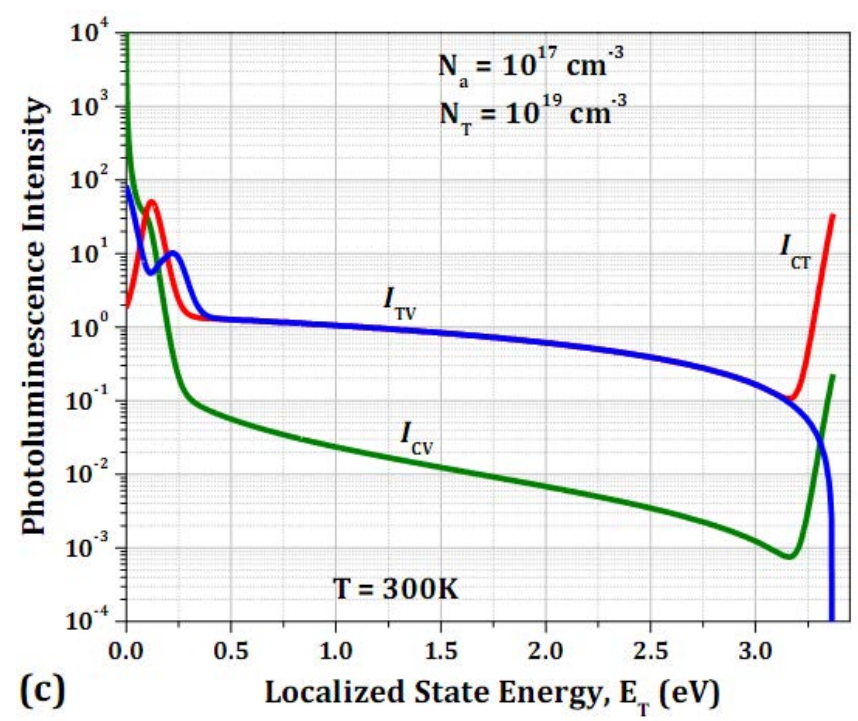

Figure 2. Variation of photoluminescence intensities with localized energies at room temperature in the bulk of $\mathrm{GaN}$, when the localized state densities are adjusted to (a) $10^{15} \mathrm{~cm}^{-3}$, (b) $10^{17} \mathrm{~cm}^{-3}$ and (c) $10^{19} \mathrm{~cm}^{-3}$. The doping level is taken to be $10^{17} \mathrm{~cm}^{-3}$ for all the samples.

\subsubsection{The case of fixed doping level, temperature, local- ized state density and varying injection levels}

For high photon energy carriers gain energies greater than their thermal energies. This reduces the rate of ionized impurity scattering as the carriers spend less time in the neighborhood of the scattering centers. If the energy levels are shallow enough for thermal excitation, the number of electrons in the conduction band increases. The net rate of electron-hole recombination is dependent on the excess carrier concentration. The rate at which holes and electrons recombine is proportional to the excess carrier concentration. The more excess carriers there are, the less time it takes for an electron and hole to meet and annihilate each other. In the $I_{C V}$ recombination with increasing illumination, the photoluminescence intensity 
increases. In the conduction band to trap level and trap level to valence band radiative recombination, photoluminescence intensity increases as illumination decreases.

The variation of illumination has its own effects on the intensity of luminescence light. Figure 3 shows that the localized state energy dependence of photoluminescence intensities due to various electronic transition levels in the $\operatorname{GaN}$ (a) when there is no illumination, (b) when illuminated with photons of $G_{0}=10^{22} \mathrm{~cm}^{-3} \mathrm{~s}^{-1}$ and (c) $G_{0}=10^{26} \mathrm{~cm}^{-3} \mathrm{~s}^{-1}$. The figure was drawn by using, doping levels and the localized state density of $10^{16} \mathrm{~cm}^{-3}$ and $10^{17} \mathrm{~cm}^{-3}$, respectively at $T=300 \mathrm{~K}$.

Figure 3 (a) illustrates that the localized state energy dependence of photoluminescence intensities when there is no illumination. When there is no illumination there is no movements of carriers and they are located in their respective bands. So, the intensity of light in the conduction band to valence radiative recombination decrease from $10^{3}$ to $10^{-2}$ and dominates only at very low energies. The intensity of light in Conduction band to localized trap level radiative recombination increases from $10^{0}$ to $10^{1}$ at low energy and decrease for the remaining energies. The intensity of light in localized trap level to Valence band radiative recombination decreases from $10^{1}$ to $10^{-3}$. It dominates from $0.25 \mathrm{eV}$ to $3.1 \mathrm{eV}$ values of localized trap energies.

Figure 3 (b) depicts that the localized state energy dependence of photoluminescence intensities at illumination of $G_{0}=10^{22} \mathrm{~cm}^{-3} \mathrm{~s}^{-1}$. The intensity of light in the conduction band to valence band radiative recombination decrease from $10^{3}$ to $10^{0}$ and again start to increase for higher energies because the conduction band electrons becomes ionized donor. It dominates for all energies. The intensity of light in Conduction band to localized trap level radiative recombination increases from $10^{-3}$ to $10^{1}$ and does not dominates for all localized energies. Due to holes traps effects, the intensity of light intensity of light in localized trap level to Valence band radiative recombination increases from $10^{1}$ to $10^{0}$. Starting from $0.25 \mathrm{eV}$ values of energy it decreases.

Figure 3 (c) depicts that the localized state energy dependence of photoluminescence intensities at illumination of $G_{0}=10^{26} \mathrm{~cm}^{-3} \mathrm{~s}^{-1}$. The intensity of light in the conduction band to valence band radiative recombination decrease from $10^{3}$ to $10^{1}$ and again start to increase for higher energies. It dominates for all energies. The intensity of light in Conduction band to localized trap level radiative recombination increases from $10^{-3}$ to $10^{-1}$. There is holes' trap effect in the intensity of light in the localized trap level to valence band radiative recombination. Due to this effect, it decreases from $10^{-1}$ to $10^{-2}$.
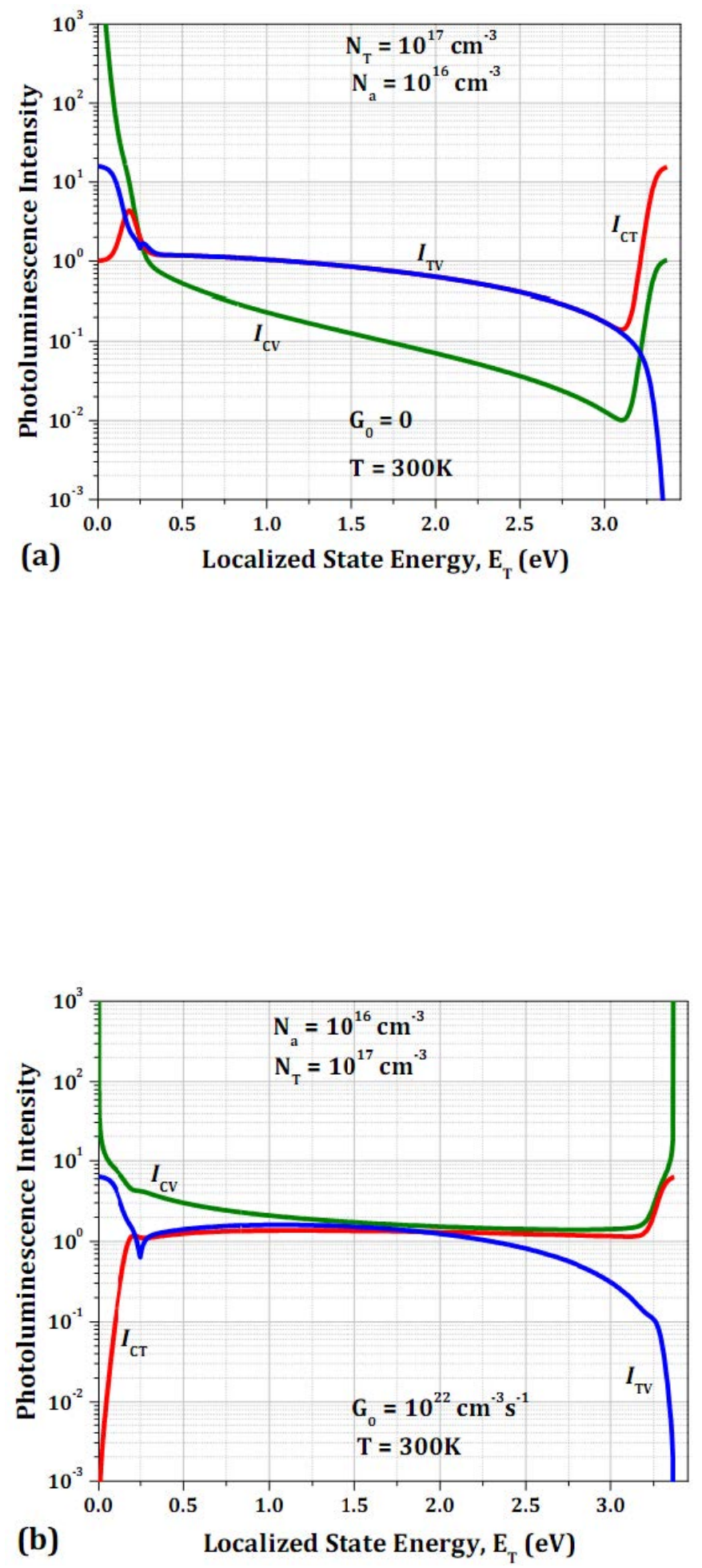


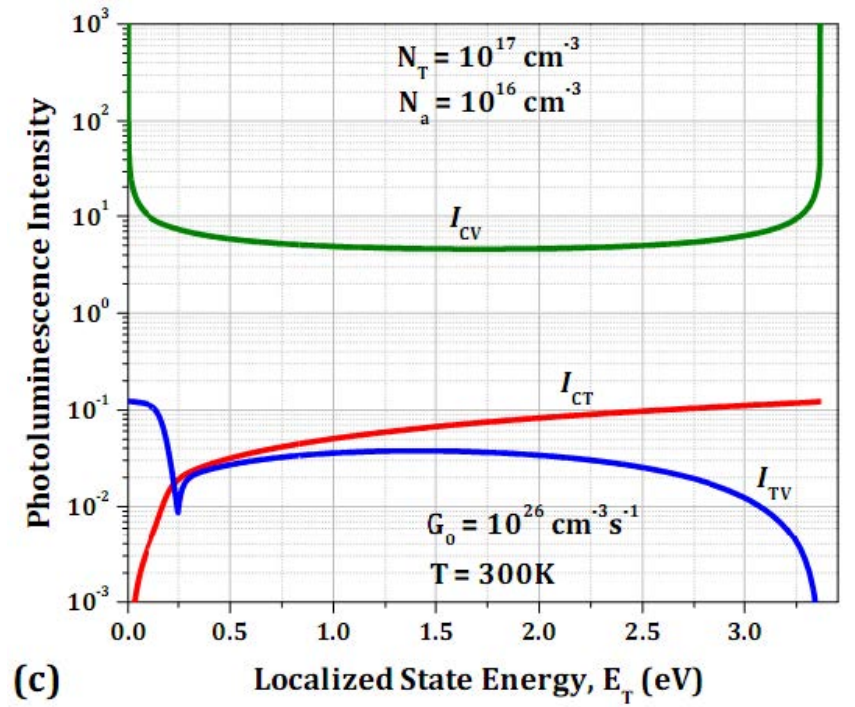

Figure 3. Localized state energy dependence of room temperature of photoluminescence intensities in the $\operatorname{GaN}$ (a) when there is no illumination, (b) when illuminated with photons of $G_{0}=10^{22} \mathrm{~cm}^{-3} \mathrm{~s}^{-1}$ and (c) $G_{0}=$ $10^{26} \mathrm{~cm}^{-3} \mathrm{~s}^{-1}$. The doping levels and the localized state density are taken to be $10^{16} \mathrm{~cm}^{-3}$ and $10^{17} \mathrm{~cm}^{-3}$, respectively for all the samples.

\section{Summary and Conclusion}

This work has discussed the photoluminescence of Gallium nitrate compound semiconductor in different radiative recombination mechanisms are analyzed by considering different factors that determines carriers density. The concentration of electrons in valence band $n_{1}$ and holes in the conduction bands $p_{1}$, are calculated based on localized trap energy. From the calculations, the concentrations of electrons in the valence band increases as trap energy increases and vice versa. Moreover, the concentration of holes in the conduction band increases with decreasing trap energy. Based on the total density of states and carries concentration, the thermal equilibrium electrons occupancy $n_{0 T}$ and thermal equilibrium holes occupancy $p_{0 T}$, are obtained at room temperature. With increasing localized trap energy level, $p_{0 T}$ increase and $n_{0 T}$ decreases.

The three radiative recombination lifetime was extracted as a function of trap energy using a steady-state carrier's density of states. The Conduction band to Valence band recombination lifetime remains constant as a function of trap energy. Conduction band to Trap level recombination lifetime decreases with increasing trap energy. The Localized Trap level to Valence band recombination lifetime decreases as localized trap energy level increases.

The net rate of electron-hole recombination is dependent on the excess carrier concentration. It was shown that as the carrier concentrations increase, the rate of recombination increases. Upon the determination of excess electrons lifetime, the net radiative recombination mechanisms in the corresponding radiative recombination mechanism are determined. It was seen that Carrier's lifetime greatly reduce the net recombination rate.

The intensity of light depends on the amount of illumination and lifetime of excess electrons in the corresponding radiative recombination mechanisms. It shows the amount of light in the bands and the more luminescent bands. Since there is no effects of traps in the conduction to valence band radiative recombination, rate of recombination is high and photoluminescence intensity of light becomes high. The intensity of light decreases with increasing localized trap energy in this band. It was shown that due to trap effects in the conduction band to trap level radiative recombination, the recombination rate reduces and hence decreases the intensity of light in the band. The intensity of light increases with increasing localized trap energy in this band. Due to the effects of trap, only small amount of electrons on localized Centre and excess of holes in the valence band. These decreases the recombination rates and hence decreases the intensity of light in the Trap level to valence band radiative recombination. Under different illumination and impurity density of states, different radiative recombination mechanisms are dominated. At low values of illumination, the intensity of light in band-to-band radiative recombination mechanism dominates only for low values of energies. But at low values of illumination, it dominates for all values of localized trap energies. For low values of impurity trap density, only $I_{C V}$ dominates and for high values of impurity trap density only $I_{C T}$ dominates for all energies.

\section{REFERENCES}

[1] Zhang R F and Veprek S 2007 Acta Materialia 554615.

[2] H. Amano, I. Akasaki, T. Kozowa, K. Hiramatsu, J. Luminescence 40-41,121 (1988).

[3] S. Nakamura and G. Fasol, The Blue Laser Diode: GaN based light emitters and lasers, Springer (1997).

[4] Bougrov V., Levinshtein M.E., Rumyantsev S.L., Zubrilov A., in Properties of Advanced SemiconductorMaterials GaN, AlN, InN, BN, SiC, SiGe . Eds. Levinshtein M.E., Rumyantsev S.L., Shur M.S., John Wiley and Sons, Inc., New York, 2001, 1-30.

[5] G. Y. Zhang, Y. Z. Tong, Z. J. Yang, S. X. Jin and Z. Z. Gan Appl. Phys. Lett. 71,3376 (1997).

[6] Barker, A.S., Ilegems, M., Infrared Lattice Vibrations and Free-Electron Dispersion in GaN, Phys. Rev. B 7 (1973) 743.

[7] A. Majid, J. Iqbal, and A. Ali, "Structural, optical and magnetic properties of $\mathrm{Ce}-\mathrm{GaN}$ based diluted magnetic semiconductor," Journal of Superconductivity and Novel Magnetism, vol. 24, no. 1-2, pp. 585-590, (2011).

[8] C. G. Van de Walle and J. Neugebauer, J. Appl. Phys. 95, 3851 (2004).

[9] Nakamura, S. Introduction to Nitride Semiconductor Blue Lasers and Light Emitting Diodes, Taylor and Francis, New York, 1999. 
[10] Smith W. "Curious effect of Light on Selenium", . s.l. : Scientific American, ,29 March 1873.

[11] Wei Fan, Hu Xu, A. L. Rosa, Th. Frauenheim and R. Q. Zhang, First-principles calculations of reconstructed [0001] ZnO nanowires, Phys. Rev. B 76 (2007)

[12] B. Monemar, Phys. Rev., B 10,676 (1974)

[13] Akasaki, I., H. Amano, J. Electrochem. Soc. 141, 8 (1994b), 2266-2271.

[14] B. L. Gel'Mont, Z .N. Sokolova, and V . B . Khalfin, "Inter band Auger recombination in Laser Structure Based on GaSb , "Sov . Phys. Semiconductors. Vol.18 ,pp. 1128 1130,1984 .
[15] G. Stollwerk, A .W. Bett, S. Keser, and O.V .Sulima, "GaAs/GaSb Tandem Concentrator Solar cell, "in Proc. 14th European PVSEC, Barcelona, Spain, 1997,pp.993998.

[16] J.J.Sumakeris,and M.J.O’Loughlin,Appl.Phys.Lett.88,052110,(200€

[17] Getu Endale, Universal Journal of Materials Science, 7(3):pp.35-41,(2019).

[18] H.Matsunami and T.Kimoto,Material Science and Enginering,R.20,125(1997). 